\title{
BMJ Global Health A holistic approach to promoting early child development: a cluster randomised trial of a group-based, multicomponent intervention in rural Bangladesh
}

\author{
Helen O Pitchik (D) , ${ }^{1}$ Fahmida Tofail, ${ }^{2}$ Mahbubur Rahman, ${ }^{3}$ Fahmida Akter, ${ }^{3}$ \\ Jesmin Sultana, ${ }^{3}$ Abul Kasham Shoab, ${ }^{3}$ Tarique Md. Nurul Huda, ${ }^{3}$ Tania Jahir, ${ }^{3}$ \\ Md Ruhul Amin, ${ }^{3}$ Md Khobair Hossain, ${ }^{3}$ Jyoti Bhushan Das, ${ }^{3}$ Esther O Chung (D) , \\ Kendra A Byrd, ${ }^{5}$ Farzana Yeasmin, ${ }^{3}$ Laura H Kwong (D) , ${ }^{6}$ Jenna E Forsyth (D) ,6 \\ Malay K Mridha, ${ }^{7}$ Peter J Winch (i) , ${ }^{8}$ Stephen P Luby (i) , ${ }^{9}$ Lia CH Fernald (i) 10
}

To cite: Pitchik HO, Tofail F, Rahman M, et al. A holistic approach to promoting early child development: a cluster randomised trial of a groupbased, multicomponent intervention in rural Bangladesh. BMJ Global Health 2021;6:e004307. doi:10.1136/ bmjgh-2020-004307

Handling editor Seye Abimbola

- Additional material is published online only. To view, please visit the journal online (http://dx.doi.org/10.1136/ bmjgh-2020-004307).

Received 26 October 2020 Revised 13 January 2021 Accepted 10 February 2021

D Check for updates

C Author(s) (or their employer(s)) 2021. Re-use permitted under CC BY. Published by BMJ.

For numbered affiliations see end of article.

Correspondence to Helen 0 Pitchik; hpitchik@berkeley.edu

\section{ABSTRACT}

Introduction In low- and middle-income countries, children experience multiple risks for delayed development. We evaluated a multicomponent, group-based early child development intervention including behavioural recommendations on responsive stimulation, nutrition, water, sanitation, hygiene, mental health and lead exposure prevention.

Methods We conducted a 9-month, parallel, multiarm, clusterrandomised controlled trial in 31 rural villages in Kishoreganj District, Bangladesh. Villages were randomly allocated to: group sessions ('group'); alternating groups and home visits ('combined'); or a passive control arm. Sessions were delivered fortnightly by trained community members. The primary outcome was child stimulation (Family Care Indicators); the secondary outcome was child development (Ages and Stages Questionnaire Inventory, ASQi). Other outcomes included dietary diversity, latrine status, use of a child potty, handwashing infrastructure, caregiver mental health and knowledge of lead. Analyses were intention to treat. Data collectors were independent from implementers.

Results In July-August 2017, 621 pregnant women and primary caregivers of children $<15$ months were enrolled (group $n=160$, combined $n=160$, control $n=301$ ). At endline, immediately following intervention completion (July-August 2018), 574 participants were assessed (group $n=144$, combined $n=149$, control $n=281$ ). Primary caregivers in both intervention arms participated in more play activities than control caregivers (age-adjusted means: group 4.22, 95\% Cl 3.97 to 4.47; combined 4.77, 4.60 to 4.96; control 3.24, 3.05 to 3.39 ), and provided a larger variety of play materials (ageadjusted means: group 3.63, 3.31 to 3.96 ; combined 3.81, 3.62 to 3.99; control 2.48, 2.34 to 2.59). Compared with the control arm, children in the group arm had higher total ASQi scores (adjusted mean difference in standardised scores: $0.39,0.15$ to 0.64 ), while in the combined arm scores were not significantly different from the control $(0.25,-0.07$ to 0.54$)$.

Conclusion Our findings suggest that group-based, multicomponent interventions can be effective at improving child development outcomes in rural Bangladesh, and that they have the potential to be delivered at scale.

\section{Key questions}

What is already known?

- Caregiving interventions that include responsive stimulation improve early child development outcomes

- Water, sanitation and hygiene (WASH), nutrition, caregiver mental health and lead exposure prevention are important contributors to early child development outcomes.

What are the new findings?

- A child stimulation intervention that includes integrated contents on WASH, nutrition, caregiver mental health and lead exposure prevention, is feasible to deliver to mixed groups of pregnant women and primary caregivers of children under 24 months of age.

- This intervention improves stimulating caregiving behaviours and child development and shows the potential for impact across multiple other risk factors for poor child development.

What do the new findings imply?

- Interventions to improve early child development that integrate components on multiple risk factors for child health and development should be considered as an alternative to siloed interventions.

- Research on the impacts of multicomponent interventions on outcomes in middle and late childhood is needed to determine if these initial effects are sustained.

Trial registration number The trial is registered in ISRCTN (ISRCTN16001234).

\section{INTRODUCTION}

Early motor, cognitive and socioemotional development affect later life outcomes, including educational attainment and economic earnings. ${ }^{1}$ In low- and 
middle-income countries (LMICs), children experience a disproportionally high burden of risk factors for delayed development when compared with children in highincome countries. An estimated one-third of 3-year-old and 4-year-old children in LMICs-80.8 million children in total-did not meet basic developmental milestones in $2010 .{ }^{2}$ Factors that promote development during early life include responsive caregiving, maternal and child nutrition, caregiver's mental health, exposure to opportunities for early learning and avoidance of infection. ${ }^{3}$

Across many different countries, cultures and contexts, caregiver-support programmes have improved short-term early child development (ECD) outcomes by encouraging responsive caregiving and stimulation through the promotion of age-appropriate caregiver-child interactions. ${ }^{4}$ Interventions addressing other risk factors for poor child development including maternal mental health, nutrition and water, sanitation and hygiene (WASH) have also been shown to improve parental investments for children, ${ }^{5}$ or ECD outcomes, ${ }^{67}$ though effects are smaller than for interventions that include responsive stimulation. Additionally, lead exposure has been associated with impaired cognitive development and can occur through exposure to contaminated turmeric and leadsoldered food storage cans. ${ }^{8-10}$ Globally up to 800 million children, mostly in LMICs, have elevated lead exposure, but interventions have not assessed the impact of lead exposure reduction on ECD outcomes. ${ }^{11}$

Integrated interventions targeting multiple risk factors have been recommended in the WHO guideline for improving ECD outcomes. ${ }^{12}$ Integration of contents on multiple components may allow for efficiencies in intervention delivery through economies of scope, and may result in more holistic changes in the early environment, resulting in improved outcomes and cost savings. For example, an integrated stimulation, nutrition and health intervention in rural China showed positive effects on multiple outcomes beyond child development, including caregiver-reported child health, nutrition and diarrhoea prevalence. ${ }^{13}$ However, the evaluation of an intervention in rural India demonstrated that it is possible that integrating many intervention components may take caregivers' focus away from stimulating caregiving practices and disperse behaviour changes across multiple domains. ${ }^{14}$

Sustainability and scalability of ECD interventions are critical to their ultimate success, and this has driven the push to explore group-based delivery mechanisms. Many responsive stimulation interventions were originally developed to be delivered in home visits, which allow for more personalised coaching and problem-solving when compared with group sessions; however, delivery at-scale may be easier to attain with groups. ${ }^{15}$ Group-based intervention delivery may also improve maternal mental health by facilitating the development of formalised social support networks in the community, and may contribute to sustained changes in community norms. ${ }^{16}$ Thus, groups may promote longer term intervention effects, an important consideration given the recent demonstrations of a fade-out of initial promising effects of scaled-up home-visiting programmes. ${ }^{17}$

In this study, we aimed to evaluate the effects of a multicomponent group-based responsive stimulation, nutrition, WASH, maternal mental health and lead exposure prevention intervention in rural Bangladesh on stimulating caregiving practices, child development and multiple other risk factors for poor child development. We tested two delivery mechanisms, one that consisted of only group sessions and one that combined group sessions and home visits. We hypothesised that the integrated multicomponent intervention would improve caregiving practices, child development and caregiver mental health through both delivery mechanisms.

\section{METHODS \\ Study design and participants}

The Research on Integrated Nutrition, ECD and WASH (RINEW) intervention was a three-arm clusterrandomised controlled trial conducted in the Katiadi and Kuliarchar subdistricts of Kishoreganj District, Bangladesh. Trial arms were (1) community group sessions (group arm), (2) alternating community group sessions and home visits (combined arm) and (3) passive control. As group sessions were community based, villages were used as the unit of randomisation to avoid spillover of intervention contents across arms.

All villages in the Katiadi and Kuliarchar subdistricts with populations between 200 and 800 households were considered for inclusion except for those in Masua union, where formative work was conducted. Villages were excluded if their basic demographic factors (ie, literacy, electricity status) were more than 1.5 standard deviations (SDs) higher or lower than district averages. This was done to decrease the probability of chance imbalances in the intervention arms at baseline, which would decrease precision of effect estimates. Remaining villages were included if they were at least $2 \mathrm{~km}$ apart. Exceptions to these criteria are described in the supplementary material (online supplemental table S1).

Eligible participants were women living in the selected villages who were in their second or third trimester of pregnancy or primary caregivers of a child under 15 months of age. All participants were eligible for all 18 intervention sessions. The pregnant woman's in-utero child, or the youngest child of the primary caregiver (for participants who were not pregnant) was considered to be the child enrolled in the RINEW study. All participants gave verbal and written informed consent before being enrolled in the study.

\section{Randomisation and masking}

Each village was a cluster, and randomisation was stratified by subdistrict. Clusters within each subdistrict were randomly allocated into one of two active intervention arms, or an oversized control arm, by an investigator at 
the University of California, Berkeley (HOP). The allocation ratio was 5:5:8 in Katiadi, and 3:3:7 in Kuliarchar for the group, combined and control arms, respectively. We used an oversized control arm to improve precision when comparing each intervention arm to the control arm. Participants were randomly selected from eligible participants in each cluster. Participants were informed of their intervention assignment following the baseline survey. Study participants and community health workers (CHWs) were not masked to intervention arm as the control arm participants were not invited to sessions with CHWs and only the combined arm included household visits. To mask data collectors to group status, they were independent from CHWs and were not made aware of the study design or intervention components. Though items from the intervention sessions (toys, books) may have been visible to data collectors, they were not made aware that these contents were part of the intervention.

\section{Intervention}

The RINEW intervention took place between September 2017 and May 2018. All participants in villages randomised to either the group or combined intervention arms were invited to attend 18 intervention sessions delivered by CHWs every 2 weeks for 9 months. The integrated multicomponent intervention curriculum was developed through a year-long piloting process. Each of the individual intervention components was developed and refined by adapting existing curricula based on field testing and feedback from community members and CHWs. ${ }^{18}$ Group sessions took $45-60 \mathrm{~min}$ and home visits took 20-25 min. Those in the group arm received 18 group sessions delivered every 2 weeks in a location close to their homestead with 3-6 pregnant women and caregiverchild dyads. Those in the combined arm received nine group sessions alternating with nine individual home visit sessions, with an intervention session every 2 weeks. Groups were assembled based on geographic proximity. The material covered was equivalent across the delivery mechanisms. In home visit sessions, facilitators discussed the age-specific recommendations presented in the group sessions that were applicable to the household. CHWs did not visit the control communities.

Each intervention session included age-specific material on responsive stimulation. For caregivers with children this portion included a brief interactive discussion about the importance of play, review of activities from previous sessions, the introduction of new developmentally appropriate games with low-cost toys made from recycled materials, a local song and activities with a simple picture book. The main aim of the stimulation component in each session was to encourage caregivers to participate in responsive caregiving and create learning opportunities through positive interaction, and to teach pregnant women how to engage in responsive stimulation with their newborn children. This intervention component was adapted from the Jamaican Reach Up Programme, ${ }^{19}$ with materials added for pregnant women and caregivers with children under 6 months of age. Each session also included material on one or more of the integrated components which included nutrition, WASH, lead exposure prevention and caregiver mental health (table 1, online supplemental table S2). A tablet application was used to guide CHWs through the agerelevant curriculum depending on who was recorded present in each session, using the CommCare software platform. Pregnant participants were encouraged to watch and learn from the activities conducted with caregiver-child dyads.

For sessions 9 and onwards, other caregivers were invited to attend sessions, with a focus on assisting with childcare during the parts of the session not focused on caregiver-child interaction. In addition, concurrently with the 15th and 16th intervention sessions fathers were invited to attend two separate group sessions with 10-12 peers. These sessions primarily focused on components that required support from household decision makers, including upgrading WASH infrastructure, purchasing lead-free food storage containers and unpolished turmeric and improving the diversity of food purchased for the household.

CHWs were 18-38 year-old women (mean 28 years) from the selected villages who had completed secondary school education. Many of the CHWs (75\%) had previously worked in education or health. CHWs received 8 days of basic training, 4 days of refresher and tablet training immediately prior to the start of the intervention and 9 additional 2-3 day trainings during the 9-month intervention. Trainings included didactic sessions, in-class practice and field practice where CHWs were given feedback and practiced observing and giving feedback to others. At least one group session (or three individual home visits) per CHW was supervised during each 2-week period. Supervisors filled out session monitoring sheets and provided feedback to CHWs.

\section{Assessments}

After enrolment, baseline data were collected on demographic information for all participants and child-related measures for children over 6 months of age. A team of university-educated enumerators who were not involved in intervention delivery conducted endline data collection during two home visits immediately following intervention completion. The first visit included assessments of the home environment, child development and maternal mental health, and enumerators received 12 days of training; the second visit included assessements of WASH, nutrition and lead and enumerators received 6 days of training. Training for both modules included interactive discussion, role play and field testing in non-intervention sites followed by interobserver reliability testing, feedback and refresher trainings. Interviews were conducted in Bengali, and data was collected using a tablet computer with CommCare software. 
Table 1 Intervention components

\begin{tabular}{|c|c|}
\hline Component & $\begin{array}{l}\text { Description of the intervention components delivered in the group and combined intervention } \\
\text { arms }\end{array}$ \\
\hline Nutrition & $\begin{array}{l}\text { Contents: This component was adapted from the WASH-Benefits intervention. }{ }^{6} \text { The } \\
\text { nutrition component included specific recommendations for each trimester of pregnancy, } \\
\text { recommendations for lactation and recommendations for the complementary feeding period. } \\
\text { Supplements: Nutritional supplements were distributed to participants depending on age and } \\
\text { child nutritional status as indicated by mid-upper arm circumference (MUAC). } \\
\text { Pregnant women and caregivers of children under } 6 \text { months of age were given multiple } \\
\text { micronutrients } \\
\text { Children with a MUAC } 11.5-12.49 \mathrm{~cm} \text { were given small-quantity lipid-based supplements } \\
\text { (SonaMoni) } \\
\text { Children over } 6 \text { months with a MUAC } 12.5 \mathrm{~cm} \text { and greater were given multiple micronutrient } \\
\text { powder (Pushtikona) } \\
\text { Children over } 6 \text { months with a MUAC under } 11.5 \mathrm{~cm} \text { were referred to a health facility (control } \\
\text { arm children were also referred) }\end{array}$ \\
\hline WASH & $\begin{array}{l}\text { This component was adapted from the WASH-Benefits intervention and included activities to coach } \\
\text { participants to identify changes they could make in their own environments. }{ }^{6} \text { Soapy water bottles } \\
\text { were provided to all households. }\end{array}$ \\
\hline Lead & $\begin{array}{l}\text { This component included teaching participants about the harms of lead and encouraging changes } \\
\text { in their household to reduce lead exposure from previously identified lead sources: turmeric and } \\
\text { lead-soldered cans. } 1036\end{array}$ \\
\hline Mental health & $\begin{array}{l}\text { This component was adapted from the thinking healthy programme. }{ }^{37} \text { Through field piloting the } \\
\text { strategies were simplified, integrated with other intervention material and incorporated behavioural } \\
\text { activation. }{ }^{18}\end{array}$ \\
\hline Targeted households & $\begin{array}{l}\text { Participants with a MUAC under } 12.5 \text { were considered at risk: } 17 \text { at-risk participants who did } \\
\text { not have access to their own hygienic latrine at baseline received WASH infrastructure (a child } \\
\text { potty, a handwashing station and a dual pit latrine). } \\
\text { Pregnant women received foot measurement sticks: participants who gave birth to a child } \\
\text { who had a foot length }<7 \mathrm{~cm} \text { received a visit from the CHW who confirmed the foot length and } \\
\text { provided (1) a session to teach the mother to provide Kangaroo Mother Care (KMC) to the baby; } \\
\text { and (2) gave the mother a KMC kit that included three KMC pouches, one hat and one pair of } \\
\text { socks. }\end{array}$ \\
\hline Toys and books & $\begin{array}{l}\text { All participants in sessions received low-cost picture books and toys made out of recycled materials } \\
\text { for children over } 6 \text { months of age }\end{array}$ \\
\hline
\end{tabular}

WASH, water, sanitation and hygiene.

\section{Outcomes}

The pre-specified primary quantitative outcome of this trial was the Family Care Indicators (FCI), a caregiver report questionnaire with an observation component used to assess stimulation in the home. ${ }^{20}$ This outcome contains two primary subscales, stimulating caregiving practices and the variety of play materials available in the home. The stimulating caregiving practices subscale has questions about the variety of stimulating caregiving activities that any adult has engaged with the child in the previous 3 days (six items). We analysed data on stimulation provided by the primary caregiver who was invited to attend the intervention sessions. The variety of play materials subscale includes observations of the variety of play materials in the home that the caregiver reported the child played with in the previous 30 days (six items). During the FCI interview caregiver responsiveness and the child's environment were observed and recorded. This observation scale includes items from the Infant Toddler Home Observation for Measurement of the Environment about caregiver responsiveness and interactions with the child and two items on the safety of the home environment (online supplemental table S3) ${ }^{21}$ The prespecified secondary outcome was child development as assessed by the Ages and Stages Questionnaire Inventory (ASQi). The ASQi is primarily a caregiver report measure used to assess attainment of milestones in the communication, gross motor, fine motor, problem-solving and personal social domains of development for children between 1 and 54 months. The ASQi includes direct assessment items for a subset (50) of the questions across five domains (online supplemental table S4). The ASQi was piloted by our study team on 60 children not included in this study sample, to ensure appropriate ranking of questions. In addition, an inventory developed following the principles of the MacArthur-Bates Communicative Development Inventories (CDI) was used to capture language development in both the expressive and receptive domains. Raw ASQi and CDI scores for each domain were internally age-standardised to the control arm using age-conditional means and SDs. Children with standardised scores over 4 were excluded. Total ASQi scores were 
created by summing raw scores across the five domains before standardising.

Other outcomes included maternal dietary diversity assessed using the Minimum Dietary Diversity for Women score, an indicator of adequate dietary diversity when at least 5 of 10 food groups are consumed in the previous 24 hours. ${ }^{22}$ Dietary diversity in young children is a similar indicator, and the cut-off for achieving the minimum is the consumption of at least 5 of 8 food groups, including breastmilk. ${ }^{23}$ Maternal depressive symptoms were measured with the 20-question Center for Epidemiologic Studies Depression scale (CES-D). Maternal depressive symptoms scores were analysed with the continuous 60-point CESD-D score. Maternal knowledge about lead was assessed by asking if respondents had ever heard of lead, and household WASH status was assessed through the observed presence of a handwashing station with water and soap or a soapy water bottle and of a clean, functional, hygienic latrine in the household.

Ongoing inter-rater reliability was conducted during the endline assessment for $4.7 \%$ of the sample $(n=27)$. Inter-rater reliabilities for the ASQi domains and the home observation subscale were high (the intraclass correlation for ASQi domains was $\geq 0.99$ for all domains except for personal social, where it was 0.93 ; for the home observation subscale the ICC was 0.92)

As a supplementary analysis, we collected data on a direct-assessment measure of child development, the Bayley Scales of Infant and Toddler Development, Third Edition (Bayley-III), for a stratified random subset of 16 villages from those that had children of both sexes in each age group ( 8 control, 4 group, 4 combined); 254 children ( $\mathrm{n}=134$ control; $\mathrm{n}=120$ intervention) were randomly selected after stratifying by age group (6-12, 13-18 and 19-24 months) and sex.

\section{Statistical analysis}

Sample size calculations were conducted for the total FCI score (range 0-13), based on a difference of 2.0 in mean total score between each intervention arm and the control arm and an SD of 3.3. ${ }^{24}$ The calculations assumed an intracluster correlation of 0.20 , power of 0.80 and type 1 error of 0.05 . With 20 participants per cluster, the sample size calculations indicated that 15 control arm villages and 8 villages in each of the intervention groups were required. The study was not powered to detect differences between the two intervention delivery methods.

All analyses were conducted according to the randomised intervention arm at enrolment (intention to treat), without considering session attendance. The primary analysis consisted of age-adjusted mean differences between the control arm and each of the group and combined intervention arms and at endline for the primary outcomes (FCI play activities and play materials subscales). Secondary analyses include mean differences (for continuous outcomes) or prevalence differences (for binary outcomes) for child development and other outcomes. Potential covariates for inclusion in adjusted models were selected based on the child development literature, and included parental education, child age and sex, household income, household wall material, household assets and the outcome of interest measured at baseline. Interviewer was also included as a potential covariate in the adjustment set for child development and observed home environment outcomes. For each outcome, covariates were prescreened using a likelihood ratio test, and all covariates with $\mathrm{p}<0.20$ were included in adjusted analyses. Adjusted analyses were done with parametric g-computation using linear regression for continuous outcomes and logistic regression for binary outcomes to generate mean differences and prevalence differences for these outcomes, respectively. ${ }^{25}$ CIs were generated with bootstrapped samples clustered by village (1000 samples). For each outcome except for the Bayley-III, two comparisons to the control arm were made, one for each intervention arm. For the Bayley-III assessment, only one comparison to the control arm was made, with children in any intervention arm combined due to small sample sizes. No adjustments were made for multiple comparisons. ${ }^{26}$ Analyses were performed in Stata V.14 and R (V.4.0.1, Vienna, Austria), with the riskCommunicator package. ${ }^{27}$

\section{Role of the funding source}

This research was funded by the Bill and Melinda Gates Foundation. The funders approved the study design, but did not play a role in data collection, analysis, or interpretation of the data, in the writing of the report, or in the decision to submit the article for publication. The corresponding author had full access to the data for this study and had final responsibility for the decision to submit for publication.

\section{Patient and public involvement}

Feedback from community members was used to adapt and refine intervention components prior to intervention delivery.

\section{RESULTS}

Between July and August 2017, fieldworkers enrolled 621 pregnant women and primary caregivers of children under 15 months of age located in 31 villages in the RINEW trial. At intervention endline, 47 (7.6\%) participants were lost to follow-up, and 6 participants had only 1 day of data collection complete, resulting in full data collection on $574(91.4 \%)$ participants (figure 1); the majority of those lost had migrated. Loss to follow-up was not statistically significantly different across study arms (control 6.6\%; group 10.0\%; combined 6.9\%), or demographic variables collected from participants at baseline. Intervention arms were similar when compared with the control arm across many baseline values (table 2). At intervention endline, the mean age of the children assessed was 16.5 months (range 3.9-26.4, SD 5.4), and the primary caregiver was the target child's mother for 


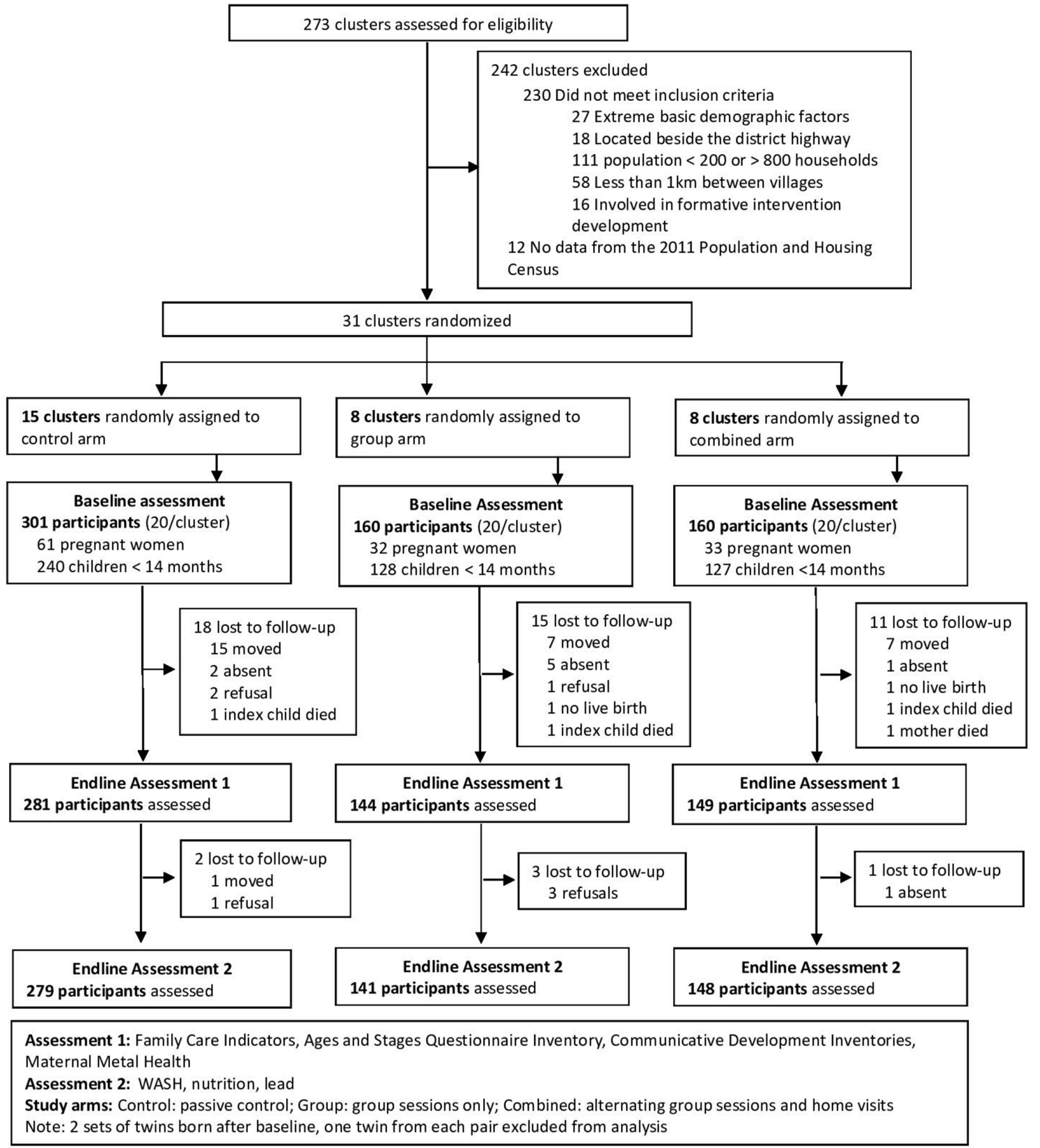

Figure 1 Trial profile.

570 (99\%) participants (4 interviews were done with other female primary caregivers of the child).

The mean number of the 18 sessions attended was similar across arms, with 14.2 (SD 4.0) in the group arm, and 15.4 (SD 3.2) in the combined arm. Participants in both intervention arms had a higher prevalence of any children's picture books in the home at intervention endline (control 19\%, group 85\%, combined 93\%), an indication that participants kept the books they received in intervention sessions.

\section{Home stimulation and child development outcomes}

Children in the group and combined intervention arms received significantly more stimulating activities in the past 3 days from their primary caregiver (age-adjusted means: group 4.22 (95\% CI 3.97 to 4.47 ); combined 4.77
(4.60 to 4.96); control 3.24 (3.05 to 3.39$)$ ), had a larger variety of stimulating play materials in the home (ageadjusted means: group 3.63 (3.31 to 3.96); combined 3.81 (3.62 to 3.99); control 2.48 (2.34 to 2.59 )) and had improved scores for the observation of caregiver responsiveness and the child's environment scale (age-adjusted means: group 8.82 (8.59 to 9.10 ); combined 8.93 (8.67 to 9.18); control 8.26 (8.05 to 8.45)) when compared with the control arm (figure 2, unadjusted means and adjusted mean differences in table 3). For comparison with other work, we calculated the unadjusted Cohen's d effect size for the stimulating caregiving activities outcome for the group (0.66 (0.45 to 0.87$))$ and combined (1.08 (0.87 to 1.29)) arms (results not shown). The stimulation activities 'played with', 'read books to' and 'sang songs to' were 
Table 2 Characteristics of the sample at baseline

\begin{tabular}{|c|c|c|c|}
\hline & \multicolumn{3}{|c|}{ Study arm, n (\%) or mean \pm SD } \\
\hline & Control $(n=301)$ & Group $(n=160)$ & Combined $(n=160)$ \\
\hline \multicolumn{4}{|l|}{ Caregiver characteristics } \\
\hline Age & $25 \pm 5.6$ & $25 \pm 6.4^{*}$ & $25 \pm 6.2$ \\
\hline Completed primary education & $173(57 \%)$ & $86(54 \%)$ & $101(63 \%)$ \\
\hline Pregnant woman enrolled & $61(20 \%)$ & $32(20 \%)$ & $33(21 \%)$ \\
\hline CES-D score (0-60) & $12.4 \pm 8.6$ & $12.8 \pm 8.7^{*}$ & $13.4 \pm 9.7$ \\
\hline Knowledge of lead & $67(22 \%)$ & $47(29 \%)$ & $49(31 \%)$ \\
\hline \multicolumn{4}{|l|}{ Child characteristics $(n=496)$} \\
\hline Age (in months) & $7.0 \pm 3.9$ & $6.7 \pm 3.9$ & $7.5 \pm 4.0$ \\
\hline Female & $134(56 \%)$ & $78(61 \%)$ & $66(52 \%)$ \\
\hline FCI Play activities subscale (0-6)‡ & $3.3 \pm 1.7$ & $3.8 \pm 1.4$ & $3.4 \pm 1.6$ \\
\hline FCI Play materials subscale (0-6)‡ & 2.1土1.1㧊 & $2.2 \pm 1.0$ & $2.2 \pm 1.2$ \\
\hline Home observation subscale $(0-11) \ddagger$ & $7.9 \pm 1.5$ & $8.1 \pm 1.4$ & $8.2 \pm 1.3$ \\
\hline $1+$ children's book(s) present in home $(n=288) \neq$ & $16(11 \%)$ & $9(14 \%) \S \S$ & $6(8 \%)$ \\
\hline MUAC <12.5 cm $(n=296) \ddagger$ & 11 (8\%)ףி & $6(8 \%) \S \S$ & $7(9 \%)$ \\
\hline \multicolumn{4}{|l|}{ Household characteristics } \\
\hline Household size & $5.2 \pm 2.2$ & $5.3 \pm 2.6$ & $5.2 \pm 2.0$ \\
\hline Number of children 2-15 years & $1.3(1.2)$ & $1.2(1.1)^{*}$ & $1.2(1.2)$ \\
\hline Has cement floor & $65(22 \%)$ & $28(18 \%)$ & $26(16 \%)$ \\
\hline Has brick walls & $74(25 \%)$ & $27(17 \%)$ & $26(16 \%)$ \\
\hline Has electricity & $243(81 \%)$ & $150(94 \%)$ & $139(87 \%)$ \\
\hline \multicolumn{4}{|l|}{ WASH } \\
\hline $\begin{array}{l}\text { Access to a handwashing station with water and soap } \\
\text { or soapy water }\end{array}$ & $62(21 \%)$ & $36(23 \%)$ & $33(21 \%)$ \\
\hline Access to a hygienic latrine§ & $102(34 \%)$ & $59(37 \%)$ & $43(27 \%)$ \\
\hline Use of potty $\ddagger$ ( $(n=297)$ & 35 (24\%)ףๆ & $16(22 \%)$ & $22(28 \%)$ \\
\hline \multicolumn{4}{|l|}{ Nutrition } \\
\hline Maternal number of food groups & $5.0 \pm 1.3$ & $4.9 \pm 1.3$ & $5.0 \pm 1.4$ \\
\hline Maternal minimum dietary diversity ${ }^{\star \star}$ & $182(60 \%)$ & $88(55 \%)$ & $101(63 \%)$ \\
\hline Child number of food groups $(n=272) \ddagger$ & $3.8 \pm 1.5$ & $3.7 \pm 1.4 \neq \ddagger$ & $4.1 \pm 1.4$ \\
\hline Child minimum dietary diversitył†† $(n=272)$ & $45(35 \%)$ & 19 (29\%)㧊 & $28(37 \%)^{\star \star \star}$ \\
\hline
\end{tabular}

${ }^{*} \mathrm{n}=159,1$ participant did not respond.

†Including index children born as of the baseline assessment.

¥Index children $>6$ months of age at baseline included $(n=296$, control=144, group=73, mixed=79), unless otherwise indicated.

$\S$ Clean, functional, Hygienic latrine. Government of Bangladesh National Sanitation Strategy, 2005 definition of hygienic latrine: Flush or pour-flush toilet/latrine to (1) Piped sewer system or (2) Septic tank; Pit latrine with slab and water seal; Pit latrine with slab and lid, no water seal; Pit latrine with slab and flap, no water seal; ventilated improved Pit latrine; composting latrine.

qUse of potty for $>50 \%$ of defecation events in last week.

${ }^{*}$ Mother reported eating 5 or more food groups in the last 24 hours, out of the following 10 groups: grains, roots and tubers, pulses, nuts and seeds, dairy products, animal flesh foods, eggs, dark green leafy vegetables, other vitamin A rich fruits and vegetables, other vegetables, other fruits.

††Children $>6$ months reported eating 5 or more food groups in the last 24 hours, out of the following groups: breast milk, grains, roots and tubers, legumes and nuts, dairy products, animal flesh foods, eggs, vitamin A rich fruits and vegetables, other fruits and vegetables.

$\ddagger \ddagger n=143$

$\S \S n=66$

ๆी $n=145$

*** $\mathrm{n}=76$

CES-D, Center for Epidemiologic Studies 20 Question Depression questionnaire, scores range from 0-60, with higher scores indicating more depressive symptoms experienced; $\mathrm{FCl}$, Family Care Indicators, the play activities subscale is a sum score of the number of play activities that the caregiver participated in with the child in the previous three days (0-6), the play materials subscale is the number of varieties of play materials observed in the home, and reported that the child played with in the last 30 days (0-6); MUAC, Mid-upper arm circumference; $\mathrm{WASH}$, water, sanitation and hygiene. 


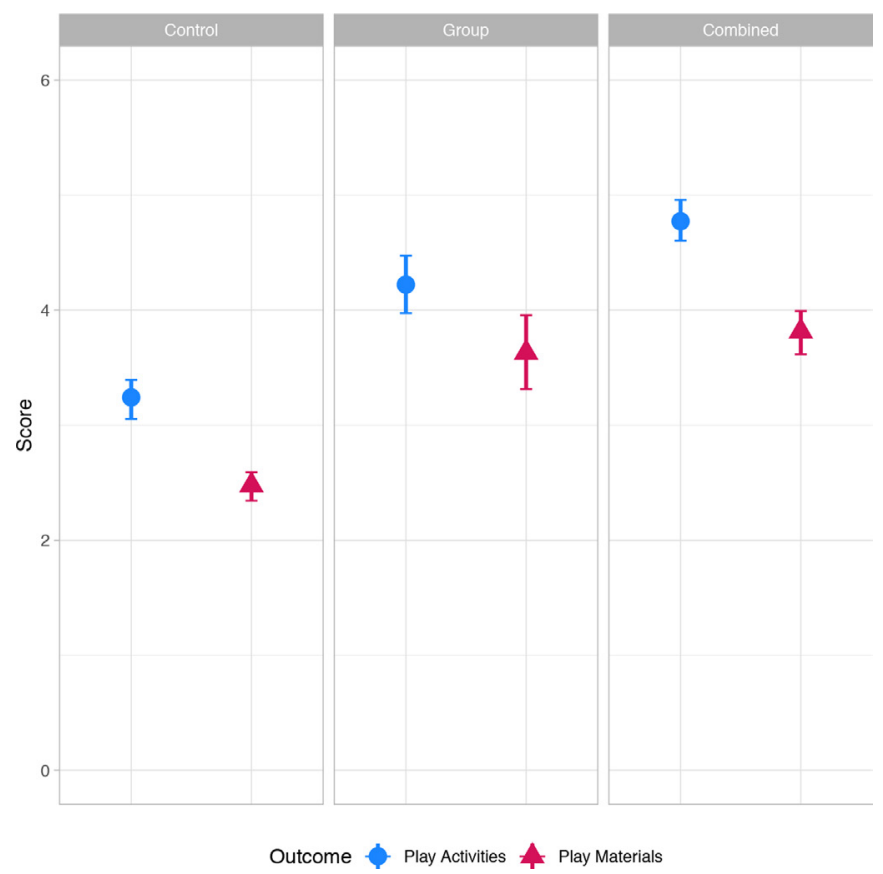

Figure 2 Mean stimulation in the home by study arm at endline. Points represent mean scores in each intervention arm, adjusted by child age at endline. Bars represent $95 \%$ Cls. Play activities (0-6): number of play activities that the primary caregiver engaged in with the child in the last 3 days. Individual items summed, and include: read books or looked at picture books; told stories; sang songs; took outside the home; played; named, counted or drew. Play materials (0-6): number of varieties of play materials observed in the home, and reported that the child played with in the last 30 days. Individual items summed, and include things: that play music; for drawing or writing; for pretending; used when running or jumping; for teaching shapes; for stacking.

the stimulation activities that had the highest prevalence differences when comparing the intervention groups to the control (online supplemental figure S1).

Children in the group and combined arms scored higher than the control arm on all domains of the ASQi and CDI assessments. Differences for the group arm were significant for all domains except for problem-solving and receptive language, and differences for the combined arm were not significant for any domains (adjusted mean differences for standardised total ASQi score: group vs control 0.39 (0.16 to 0.64 ); combined vs control 0.25 $(-0.07$ to 0.54$)$ ) (figure 3 , online supplemental table S5). In the supplementary analysis comparing both intervention arms to the control arm for the five domains of the Bayley-III assessment, the expressive communication and total Bayley-III scores were significantly higher for children in any intervention group compared to control (adjusted mean differences: expressive communication 0.33 (0.02 to 0.64 ); total Bayley-III score 0.38 (0.06 to 0.74; online supplemental figure S2).

\section{Maternal mental health, nutrition, WASH and lead outcomes}

In both intervention arms, participants reported fewer depressive symptoms as compared with the control arm, indicated by lower CES-D scores, with a control arm mean of 15.01 (SD 8.96). The adjusted mean differences were significant for the group arm $(-2.05$ (95\% CI -3.23 to 0.66$)$ ), but not the combined arm $(-1.34(-3.12$ to 0.41 ); table 3 ). Minimum dietary diversity for mothers and children was improved in the combined intervention arm with adjusted prevalence differences of 0.14 (0.04 to 0.22$)$ for mothers and 0.14 (0.05 to 0.19$)$ for children. There were no significant differences between the group and control arms for maternal or child dietary diversity. There was no difference in the presence of a functional, clean and hygienic latrine for either intervention arm when compared with the control, and only the group arm had higher prevalence of a handwashing station with soap and water (adjusted prevalence difference: 0.12 (0.01 to 0.24$)$ ). Participants in both intervention arms had greater potty use when compared with the control, with differences significant for the combined arm (adjusted prevalence differences: combined 0.10 (0.00 to 0.21$)$; group 0.13 (-0.02 to 0.28$)$ ). Knowledge of lead was significantly higher in both intervention arms, with a control arm prevalence of 0.24 and adjusted prevalence differences of 0.51 ( 0.41 to 0.61$)$ and 0.52 (0.39 to 0.63 ) for the group and combined arm, respectively.

\section{DISCUSSION}

We found beneficial intervention impacts on our primary outcome of stimulation in the home, our secondary outcome of child development, as well as across a range of risk factors for child development addressed by the multicomponent intervention including caregiver depressive symptoms, caregiver and child dietary diversity, WASH and knowledge of lead. We observed impacts on play activities, play materials and observations of the home environment in both intervention arms. However, as the source of each play material was not asked, the results cannot be disaggregated by materials provided in the sessions and materials caregivers obtained on their own. Child development scores, as assessed by the ASQi and CDI were higher in both intervention arms when compared with the control, with differences for 6 of the 7 domains significant for the group arm, but not for the combined arm. Differences in standardised child development scores for the group intervention arm are between 0.18 to 0.39 , similar to those from a group intervention in rural India which found significant improvements in cognition scores of $0.28 \mathrm{SDs},{ }^{28}$ and an integrated home visiting programme in rural China, with intervention effects of 0.24 SDs. ${ }^{13}$ The results from a supplementary analysis on a subset of participants demonstrate improved receptive communication and total Bayley-III scores among those who received any intervention compared with the control. Though the current study was not powered to compare the group and combined arms directly, recent work from rural India finds similar effects on child development outcomes from group and individual home-based sessions. ${ }^{28}$ Further, recent 
Table 3 Child development, maternal mental health, nutrition, water, sanitation and hygiene and lead outcomes at endline

\begin{tabular}{|c|c|c|c|c|c|}
\hline \multirow[b]{2}{*}{ Indicators } & \multicolumn{3}{|c|}{ Unadjusted mean \pm SD or $\mathbf{N}(\%)$ by arm } & \multicolumn{2}{|c|}{$\begin{array}{l}\text { Adjusted mean difference or risk difference } \\
\text { versus control arm }(95 \% \mathrm{Cl})\end{array}$} \\
\hline & Control & Group & Combined & Group & Combined \\
\hline \multicolumn{6}{|l|}{$\mathrm{FCl}$} \\
\hline Activities & $3.2 \pm 1.5$ & $4.2 \pm 1.5$ & $4.8 \pm 1.3$ & $1.05(0.72$ to 1.34$)$ & $1.56(1.33$ to 1.78$)$ \\
\hline Materials & $2.5 \pm 1.4$ & $3.64 \pm 1.7$ & $3.9 \pm 1.6$ & 1.18 (0.88 to 1.51$)$ & 1.36 (1.18 to 1.51$)$ \\
\hline Observation & $8.3 \pm 1.5$ & $8.8 \pm 1.2$ & $8.9 \pm 1.5$ & $0.56(0.26$ to 0.89$)$ & 0.67 (0.35 to 0.99$)$ \\
\hline \multicolumn{6}{|l|}{ ASQi } \\
\hline Communication & $56.0 \pm 16.4$ & $58.4 \pm 15.3$ & $58.6 \pm 16.3$ & $0.32(0.10$ to 0.57$)$ & $0.21(-0.04$ to 0.49$)$ \\
\hline Fine motor & $50.2 \pm 11.3$ & $51.8 \pm 10.1$ & $51.6 \pm 11.6$ & $0.36(0.11$ to 0.63$)$ & $0.23(-0.04$ to 0.49$)$ \\
\hline Gross motor & $58.6 \pm 15.1$ & $60.8 \pm 14.1$ & $59.3 \pm 16.1$ & 0.27 (0.13 to 0.44$)$ & $0.04(-0.18$ to 0.22$)$ \\
\hline Problem-solving & $55.6 \pm 15.8$ & $56.9 \pm 14.6$ & $58.2 \pm 15.6$ & $0.18(-0.04$ to 0.43$)$ & $0.19(-0.14$ to 0.49$)$ \\
\hline Personal social & $55.3 \pm 16.3$ & $57.7 \pm 15.5$ & $58.0 \pm 16.4$ & $0.34(0.10$ to 0.63$)$ & $0.30(-0.04$ to 0.64$)$ \\
\hline Total & $275.7 \pm 71.2$ & $284.6 \pm 65.2$ & $285.0 \pm 72.6$ & 0.39 (0.16 to 0.64$)$ & $0.25(-0.07$ to 0.54$)$ \\
\hline \multicolumn{6}{|l|}{ CDI } \\
\hline Receptive & $44.8 \pm 23.7$ & $49.0 \pm 22.4$ & $49.0 \pm 23.3$ & $0.25(-0.04$ to 0.55$)$ & $0.19(-0.15$ to 0.52$)$ \\
\hline Expressive & $16.8 \pm 17.2$ & $18.9 \pm 17.9$ & $19.2 \pm 18.3$ & $0.29(0.06$ to 0.50$)$ & $0.17(-0.17$ to 0.53$)$ \\
\hline \multicolumn{6}{|l|}{ Depressive symptoms } \\
\hline CES-D score & $15.0 \pm 9.0$ & $13.2 \pm 7.0$ & $14.1 \pm 9.1$ & $-2.06(-3.23$ to -0.66$)$ & $-1.34(-3.12$ to 0.41$)$ \\
\hline \multicolumn{6}{|l|}{ Minimum dietary diversity } \\
\hline Child* & $146(54 \%)$ & $85(61 \%)$ & $98(68 \%)$ & $0.07(-0.03$ to 0.17$)$ & 0.14 (0.04 to 0.22$)$ \\
\hline Maternal† & $177(63 \%)$ & $89(63 \%)$ & $113(76 \%)$ & $0.03(-0.07$ to 0.12$)$ & 0.14 (0.05 to 0.19$)$ \\
\hline \multicolumn{6}{|l|}{ WASH } \\
\hline Hygienic latrineł & $96(34 \%)$ & $49(35 \%)$ & $51(35 \%)$ & $-0.03(-0.19$ to 0.11$)$ & $0.02(-0.12$ to 0.15$)$ \\
\hline $\begin{array}{l}\text { Handwashing station with soap } \\
\text { and water }\end{array}$ & $59(21 \%)$ & $44(31 \%)$ & $36(24 \%)$ & 0.12 (0.01 to 0.24$)$ & $0.04(-0.08$ to 0.19$)$ \\
\hline Use of potty§ & $55(20 \%)$ & $42(30 \%)$ & $44(30 \%)$ & $0.13(-0.02$ to 0.28$)$ & 0.10 (0.00 to 0.21$)$ \\
\hline \multicolumn{6}{|l|}{ Lead } \\
\hline Knowledge of lead & $68(24 \%)$ & $103(73 \%)$ & $110(74 \%)$ & 0.51 (0.40 to 0.60$)$ & 0.52 (0.39 to 0.63$)$ \\
\hline
\end{tabular}

Activities: number of play activities that the mother participated in with the child in the last three days (out of six). Materials: number of varieties of play materials observed available in the home (out of six). Observation: 11 observation items about caregiver-child interactions during the interview, and observations of the home environment (S5 for details)

For ASQi and CDI results: unadjusted mean values are raw values before standardisation, all adjusted mean differences use scores which are internally age-standardised to the control arm, and point estimates represent SDs from the control arm mean.

Adjusted analyses include the following potential covariates: interviewer (for FCl, ASQi and CDI outcomes), maternal and paternal education, child age, child sex, household income above the median, household wall material, presence of electricity in the home, the presence of household assets (wardrobe, table, chair, watch/clock, television, bicycle, sewing machine) and the measure assessed at baseline (if assessed in the whole population). Covariates with $p<0.20$ from a likelihood ratio test for each outcome are included in adjusted analyses. $\mathrm{n}$ for ASQi and CDI outcomes (excluding outliers \pm 4 SD from the control arm mean): communication $n=566$; fine motor $n=559$, gross motor $n=563$; problem-solving $n=563$; personal social $n=550$; total $n=532$; receptive $n=573$; expressive $n=498$ (only children over 9 months of age included).

${ }^{*}$ Children $>6$ months $(n=555)$ reported eating 5 or more food groups in the last 24 hours, out of the following groups: breast milk, grains, legumes, dairy products, flesh foods, eggs, vitamin A rich fruits and vegetables, other fruits and vegetables $(n=553)$.

†Mother reported eating 5 or more food groups in the last 24 hours, out of the following 10 groups: grains, legumes, nuts and seeds, dairy products, flesh foods, eggs, vitamin A rich fruits and vegetables, other vitamin A rich fruits and vegetables, other vegetables, other fruits. ‡Hygienic latrine (according to Government of Bangladesh National Sanitation Strategy 2005): Flush or pour-flush toilet/latrine to (1) piped sewer system, (2) septic tank; pit latrine with slab and water seal; pit latrine with slab and lid, no water seal; pit latrine with slab and flap, no water seal; ventilated improved pit latrine; composting latrine.

§Use of potty for $>50 \%$ of defecation events for the index child in the last 7 days.

ASQi, Ages and Stages Questionnaire Inventory; CDI, Communicative Development Inventories; CES-D, Center for Epidemiologic Studies 20 question Depression scale; WASH, Water, Sanitation and Hygiene. 


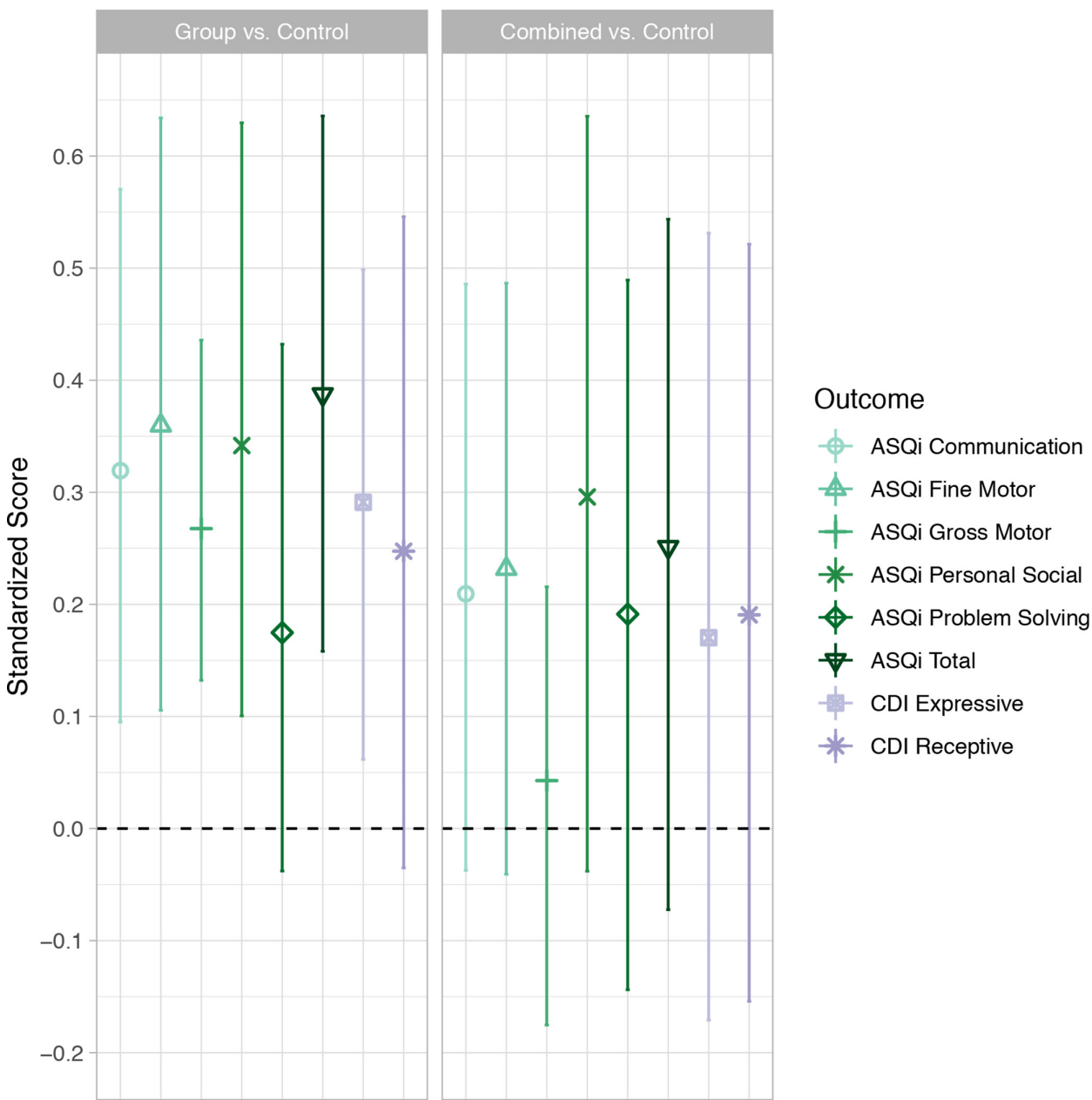

Figure 3 Adjusted mean differences in age-standardised Ages and Stages Questionnaire Inventory (ASQi) and Communicative Development Inventory (CDI), by intervention arm. Results for all domains are internally age-standardised to the control arm, points represent mean differences in standardised scores between each intervention arm and the control arm, lines represent $95 \%$ Cls. $n$ by domain, after removing outliers and missing data: ASQi communication, $n=566$; ASQi fine motor, $n=559$; ASQi gross motor, $n=563$; ASQi problem-solving, $n=563$; ASQi personal social, $n=550$; ASQi total, $n=532$; CDI receptive $\mathrm{n}=573$; CDI expressive $\mathrm{n}=498$ (only including children over 9 months old).

work from rural Kenya indicates that group sessions may outperform combined delivery in some settings. ${ }^{29}$ Our work supports the delivery of multicomponent, mixed-age group sessions to improve risk factors for poor child development in rural Bangladesh.

Maternal depression is a risk factor for delayed child development, and has been associated with poor behavioural and developmental outcomes. ${ }^{30}$ We found fewer reported depressive symptoms from caregivers in both intervention arms, with a significant difference for the group arm. We hypothesise that the focus on maternal depression and the social support facilitated during the group intervention sessions contributed to the reduced depressive symptoms in both intervention arms. The effect may have been stronger in the group arm because this delivery mode offered structured peer social support 18 times over the course of the intervention compared with nine times in the combined arm. A meta-analysis of the effect of child stimulation interventions on caregiver depressive symptoms found no significant effect $(-0.20(-0.23$ to 0.03$)) .{ }^{31}$ However, the only group-based intervention that included contents on mental health found an effect size of $-0.54(-0.76$ to -0.32$))$. This is higher in magnitude than the effect in the group arm of the current study (unadjusted Cohen's d: -0.22 ( -0.42 to $-0.02)) .^{32}$

We found improvements in nutrition, WASH and knowledge of lead in the intervention group. In the combined arm, a higher proportion of children and caregivers had a more diverse diet, though no difference was found for the group arm. As improving dietary diversity require changes in both purchasing and meal preparation, it may be that an approach where CHWs can respond to individual needs of families is required. There was no difference in presence of hygienic latrines in either intervention arm when compared with the control, more families in the group arm had a handwashing station with soap and water, and more caregivers in both arms reported that their child used a potty regularly, though the difference was only statistically significant for the combined arm. We do not know if the 17 families who were provided a potty would have purchased and used one in the absence of 
it being provided. We hypothesise that a subsidy may be required to improve hygienic latrine status in this lowincome community given the investment required to upgrade WASH infrastructure. Caregiver's knowledge of lead was improved in both arms, with large effect sizes. Lead is an invisible toxin unknown to the majority of the population at baseline, thus knowledge of lead is the first step towards reducing exposure. Secondary analyses will further investigate the intervention effects on behaviours related to lead exposure.

The unadjusted Cohen's d effect sizes for stimulating caregiving practices in both intervention arms (group 0.66 (95\% CI 0.45 to 0.87 ); combined 1.08 (0.87 to 1.29)) are slightly larger than the pooled effect sizes (0.57 (0.37 to 0.77$))$ from a recent meta-analysis of the effect of stimulation interventions on stimulation in the home, measured by the FCI and Infant Toddler Home Observation for Measurement of the Environment. ${ }^{31}$ The interventions in the meta-analysis included at most two additional components in additional to child stimulation, whereas our study included four additional components. Further, none of the interventions in the meta-analysis were delivered to both pregnant women and caregivers of children under 24 months of age. Thus, our findings suggest that the effects on caregiver-related outcomes were not diminished with the inclusion of multiple integrated intervention components, nor intervention delivery across both pregnant women and caregivers of young children.

In the RINEW intervention, groups were based on geographical proximity to reduce barriers to attendance, and included pregnant women and mixed-age, caregiverchild dyads. Other group-based ECD interventions are delivered to groups with children of similar ages, to allow for the presentation of age-specific materials relavent to the whole group. ${ }^{13}$ In addition to reducing barriers to attendance, grouping participants with others who they may interact with daily may increase the potential for continued social support for intervention activities outside sessions. However, delivering sessions to a mixed-age group may increase session duration, and include less engaging components for some participants (ie, pregnancy contents for non-pregnant participants). ${ }^{33}$ In future work, the tradoffs of mixed-age delivery and geographic proximity should take into account the geographic density of eligible participants and accessability of session locations.

This study has several strengths, including the focus on a group-based intervention to address multiple risk factors for poor ECD, which makes this approach more scalable than one-on-one home visiting programmes. In addition, the intervention was delivered simultaneously to both pregnant and lactating women with children of mixed-ages, an approach that is easier to scale than more narrowly focused programmes. We used a tablet application to facilitate session delivery, which enabled the inclusion of multiple age-specific intervention components. Another unique feature of this programme was the integration of information on the reduction of heavy metal exposure, in addition to standard messages about nutrition, health and hygiene. Finally, we included a set of outcomes that spans a broad range of influences in early life and development in order to gain a more comprenensive understanding of the impact of the intervention on a child's development and well-being.

The current study has important limitations. First, due to budget constraints, the sample was not powered to detect small differences on many of the secondary outcomes, or differences between the two active intervention arms. As such, we are only able to interpret the direction and magnitude of these effects. Second, the FCI, ASQi, CDI, dietary diversity, depressive symptoms and knowledge of lead assessments are primarily based on caregiver-report, allowing for the possibility that caregiver responses about behaviours could be influenced by knowledge and social desirability, or caregivers' mental health status. The risk of respondent bias was minimised through extensive training of survey enumerators, the use of direct-observation items within the ASQi, an observation scale to complement the FCI and follow-up questions to confirm reported lead knowledge. We found significant improvements in observed caregiver responsiveness and the caregiving environment, highlighting that changes were found for observed behaviour in addition to caregiver report. Additionally, as this was the first time implementing such an intervention curriculum in Bangladesh, there were some adjustments to the strategies used to build group cohesiveness and encourage attendance, and intervention modules were refined as the sessions progressed. The results do not represent the impacts of the intervention that may be achieved with further refinements, and the current estimates may be a lower bound on the possible impact. Finally, we were not able to examine the cost effectiveness of group compared with individual or combined delivery mechanisms for integrated interventions.

This intervention illustrates the feasibility of locally recruited CHWs delivering a group-based, mixed-age, multicomponent ECD intervention in rural Bangladesh. The feasibility of scaling such a group-based intervention through a government health system, or the large-scale implementation through a regional or national nongovernment organisation is not known, and warrants exploration. A promising, recent study found that child stimulation sessions delivered through Government of Bangladesh community clinics to pairs of motherchild dyads, had large impacts on child development. ${ }^{34}$ Notably, these clinics serve as regular point of care, routinely providing maternal and neonatal healthare as well as nutrition and health education. Differences in CHW workload, session attendance and intervention impacts with each of the delivery mechanisms will inform the design of scalable and impactful child development interventions.

The long-term impact of the RINEW intervention, or similar integrated interventions targeting multiple 
risk factors for ECD, will be critical to understand the scope of intervention impact. Although many stimulation interventions have shown impacts on child development outcomes at intervention endline, there is mixed evidence on the later impacts of these early interventions. ${ }^{1735}$ It is possible that integrated interventions addressing multiple risk factors may contribute to sustained intervention impacts on child development as they more holistically improve children's early-life caregiving and health environments. Medium and long-term follow-up of children enrolled in multicomponent interventions is required to examine this hypothesis.

\section{CONCLUSION}

In conclusion, we found that a carefully designed group-based multicomponent intervention delivered by well-trained $\mathrm{CHWs}$ can address multiple additional risk factors for child development beyond stimulating caregiving, and demonstrate similar effects on stimulating caregiving as interventions with fewer integrated components. CHWs were able to deliver the complex multicomponent RINEW intervention for 9 months and community members regularly attended intervention sessions regardless of delivery platform. This multicomponent approach may be used as a template to design a scalable and impactful intervention to improve child well-being in low-income settings.

\author{
Author affiliations \\ ${ }^{1}$ Division of Epidemiology, School of Public Health, University of California, Berkeley, \\ Berkeley, California, USA \\ ${ }^{2}$ Nutrition and Clinical Services Division, International Centre for Diarrhoeal Disease \\ Research Bangladesh, Dhaka, Dhaka District, Bangladesh \\ ${ }^{3}$ Infectious Diseases Division, International Centre for Diarrhoeal Disease Research \\ Bangladesh, Dhaka, Dhaka District, Bangladesh \\ ${ }^{4}$ Department of Epidemiology, Gillings School of Public Health, University of North \\ Carolina at Chapel Hill, Chapel Hill, North Carolina, USA \\ ${ }^{5}$ WorldFish, Bayan Lepas, Penang, Malaysia \\ ${ }^{6}$ Woods Institute for the Environment, Stanford University, Stanford, California, USA \\ ${ }^{7}$ Center for Non-communicable Diseases and Nutrition, BRAC James P Grant \\ School of Public Health, BRAC University, Dhaka, Dhaka District, Bangladesh \\ ${ }^{8}$ International Health, Johns Hopkins Bloomberg School of Public Health, Baltimore, \\ Maryland, USA \\ ${ }^{9}$ Division of Infectious Diseases and Geographic Medicine, Stanford University, \\ Stanford, California, USA \\ ${ }^{10}$ Division of Community Health Sciences, School of Public Health, University of \\ California, Berkeley, Berkeley, California, USA
}

Acknowledgements The authors thank the participants, data collectors and field staff for their dedication to this study.

Contributors The research protocol was drafted by SPL with input from PJW, LCHF, FT, MKM, JEF and MR. All authors contributed to intervention design. The intervention piloting and implementation, and data collection was overseen by FT, FA, JS, AKS, TMNH, MR, TJ, MRA, MKH and JBD. The statistical approach was developed by HOP with input from SPL, FT, LCHF and TMNH. Data curation and cleaning was done by JS, AKS and HOP with input from TMNH and KAB. HOP conducted the data analysis, constructed the tables and figures, and drafted the manuscript. HOP, LCHF, SPL, PJW, FT, TMNH and MKM, contributed to interpretation of results. All authors approved of the final version of the manuscript. HOP had full access to all the data in the study and the final responsibility for the decision to submit for publication.

Funding This study was supported by a grant from the Bill and Melinda Gates Foundation (OPP1146808). icddr,b acknowledges the Bill and Melinda Gates
Foundations for funding this project. icddr,b is also grateful to the Government of Bangladesh, Canada, Sweden and UK for providing core/unrestricted support.

Competing interests None declared.

Patient consent for publication Not required.

Ethics approval Ethical approval was obtained from the International Centre for Diarrhoeal Disease Research, Bangladesh (icddr,b), and the University of California, Davis.

Provenance and peer review Not commissioned; externally peer reviewed.

Data availability statement Data are available upon request. The deidentified data collected for this study will be available from the corresponding author on request 5 years following intervention completion, or following the acceptance of all planned manuscripts. Data will be shared for research purposes only, following the receipt of a signed data-sharing agreement.

Supplemental material This content has been supplied by the author(s). It has not been vetted by BMJ Publishing Group Limited (BMJ) and may not have been peer-reviewed. Any opinions or recommendations discussed are solely those of the author(s) and are not endorsed by BMJ. BMJ disclaims all liability and responsibility arising from any reliance placed on the content. Where the content includes any translated material, BMJ does not warrant the accuracy and reliability of the translations (including but not limited to local regulations, clinical guidelines, terminology, drug names and drug dosages), and is not responsible for any error and/or omissions arising from translation and adaptation or otherwise.

Open access This is an open access article distributed in accordance with the Creative Commons Attribution 4.0 Unported (CC BY 4.0) license, which permits others to copy, redistribute, remix, transform and build upon this work for any purpose, provided the original work is properly cited, a link to the licence is given, and indication of whether changes were made. See: https://creativecommons.org/ licenses/by/4.0/.

\section{ORCID iDs}

Helen 0 Pitchik http://orcid.org/0000-0002-5665-0884

Esther 0 Chung http://orcid.org/0000-0002-2866-949X

Laura H Kwong http://orcid.org/0000-0002-9776-9355

Jenna E Forsyth http://orcid.org/0000-0003-1888-3967

Peter J Winch http://orcid.org/0000-0001-8569-5507

Stephen P Luby http://orcid.org/0000-0001-5385-899X

Lia CH Fernald http://orcid.org/0000-0003-1555-4607

\section{REFERENCES}

1 Peet ED, McCoy DC, Danaei G, et al. Early childhood development and schooling attainment: longitudinal evidence from British, Finnish and Philippine birth cohorts. PLoS One 2015;10:e0137219.

2 McCoy DC, Peet ED, Ezzati M, et al. Early childhood developmental status in low- and middle-income countries: national, regional, and global prevalence estimates using predictive modeling. PLoS Med 2016;13:e1002034.

3 Black MM, Walker SP, Fernald LCH, et al. Early childhood development coming of age: science through the life course. Lancet 2017;389:77-90.

4 Aboud FE, Yousafzai AK. Global health and development in early childhood. Annu Rev Psychol 2015;66:433-57.

5 Baranov V, Bhalotra S, Biroli P, et al. Maternal Depression, Women's Empowerment, and Parental Investment: Evidence from a Randomized Controlled Trial. Am Econ Rev 2020;110:824-59.

6 Tofail F, Fernald LC, Das KK, et al. Effect of water quality, sanitation, hand washing, and nutritional interventions on child development in rural Bangladesh (wash benefits Bangladesh): a cluster-randomised controlled trial. Lancet Child Adolesc Health 2018;2:255-68.

7 Prado EL, Larson LM, Cox K, et al. Do effects of early life interventions on linear growth correspond to effects on neurobehavioural development? A systematic review and metaanalysis. Lancet Glob Health 2019;7:e1398-413.

8 Bellinger D, Leviton A, Waternaux C, et al. Longitudinal analyses of prenatal and postnatal lead exposure and early cognitive development. N Engl J Med 1987;316:1037-43.

9 Forsyth JE, Saiful Islam M, Parvez SM, et al. Prevalence of elevated blood lead levels among pregnant women and sources of lead exposure in rural Bangladesh: a case control study. Environ Res 2018;166:1-9. 
10 Forsyth JE, Nurunnahar S, Islam SS, et al. Turmeric means "yellow" in Bengali: Lead chromate pigments added to turmeric threaten public health across Bangladesh. Environ Res 2019;179:108722.

11 UNICEF and Pure Earth. The toxic truth, 2020. Available: https:// www.unicef.org/reports/toxic-truth-childrens-exposure-to-leadpollution-2020 [Accessed 10 Jan 2021].

12 Improving early childhood development: WHO guideline. Available: https://www.who.int/publications-detail/improving-early-childhooddevelopment-who-guideline [Accessed 7 Mar 2020].

13 Luo R, Emmers D, Warrinnier N, et al. Using community health workers to deliver a scalable integrated parenting program in rural China: a cluster-randomized controlled trial. Soc Sci Med 2019;239:112545.

14 Vazir S, Engle P, Balakrishna N, et al. Cluster-Randomized trial on complementary and responsive feeding education to caregivers found improved dietary intake, growth and development among rural Indian toddlers. Matern Child Nutr 2013;9:99-117.

15 Radner JM, Ferrer MJS, McMahon D, et al. Practical considerations for transitioning early childhood interventions to scale: lessons from the saving brains portfolio. Ann N Y Acad Sci 2018;1419:230-48.

16 Tripathy $\mathrm{P}$, Nair N, Barnett S, et al. Effect of a participatory intervention with women's groups on birth outcomes and maternal depression in Jharkhand and Orissa, India: a cluster-randomised controlled trial. Lancet 2010;375:1182-92.

17 Andrew A, Attanasio O, Fitzsimons E, et al. Impacts 2 years after a scalable early childhood development intervention to increase psychosocial stimulation in the home: a follow-up of a cluster randomised controlled trial in Colombia. PLoS Med 2018;15:e1002556.

18 Akter F, Rahman M, Pitchik HO, et al. Adaptation and integration of psychosocial stimulation, maternal mental health and nutritional interventions for pregnant and lactating women in rural Bangladesh. Int J Environ Res Public Health 2020;17. doi:10.3390/ ijerph17176233. [Epub ahead of print: 27 Aug 2020].

19 Grantham-McGregor S, Smith JA. Extending the Jamaican early childhood development intervention. J Appl Res Child Informing Policy Child Risk 2016;7:4.

20 Hamadani JD, Tofail F, Hilaly A, et al. Use of family care indicators and their relationship with child development in Bangladesh. $J$ Health Popul Nutr 2010;28:23-33.

21 Bradley RH, Caldwell BM. The home inventory and family demographics. Dev Psychol 1984;20:315-20.

$22 \mathrm{FAO}$ and FHI 360. Minimum dietary diversity for women a guide to measurement. Rome, Italy, 2016.

23 World Health Organization. Operational guidance for tracking progress in meeting targets for 2025, 2017. Available: https:// www.who.int/nutrition/publications/operational-guidance-GNMFindicators/en/ [Accessed 25 Nov 2019].

24 Tofail F, Hamadani JD, Mehrin F, et al. Psychosocial stimulation benefits development in nonanemic children but not in anemic, irondeficient children. J Nutr 2013;143:885-93.
25 Ahern J, Hubbard A, Galea S. Estimating the effects of potential public health interventions on population disease burden: a stepby-step illustration of causal inference methods. Am J Epidemiol 2009;169:1140-7.

26 Rothman KJ. No adjustments are needed for multiple comparisons. Epidemiology 1990;1:43-6.

27 Grembi J, McQuade ER. riskCommunicator: G-Computation to estimate interpretable epidemiological effects, 2020. Available: https://CRAN.R-project.org/package=riskCommunicator [Accessed 22 Aug 2020].

28 Grantham-McGregor S, Adya A, Attanasio O, et al. Group sessions or home visits for early childhood development in India: a cluster RCT. Pediatrics 2020;146. doi:10.1542/peds.2020-002725. [Epub ahead of print: 04 Nov 2020].

29 Luoto JE, Lopez Garcia I, Aboud FE, et al. Group-Based parenting interventions to promote child development in rural Kenya: a multiarm, cluster-randomised community effectiveness trial. Lancet Glob Health 2020. doi:10.1016/S2214-109X(20)30469-1. [Epub ahead of print: 17 Dec 2020] (Published Online First: 17 December 2020).

30 Herba CM, Glover V, Ramchandani PG, et al. Maternal depression and mental health in early childhood: an examination of underlying mechanisms in low-income and middle-income countries. Lancet Psychiatry 2016;3:983-92.

31 Jeong J, Pitchik HO, Yousafzai AK. Stimulation interventions and parenting in low- and middle-income countries: a meta-analysis. Pediatrics 2018;141:e20173510.

32 Singla DR, Kumbakumba E, Aboud FE. Effects of a parenting intervention to address maternal psychological wellbeing and child development and growth in rural Uganda: a community-based, cluster randomised trial. Lancet Glob Health 2015;3:e458-69.

33 Yeasmin F, Winch PJ, Hwang ST, et al. Exploration of attendance, active participation, and behavior change in a group-based responsive stimulation, maternal and child health, and nutrition intervention. Am J Trop Med Hyg 2021. doi:10.4269/ajtmh.20-0991

34 Hamadani JD, Mehrin SF, Tofail F, et al. Integrating an early childhood development programme into Bangladeshi primary healthcare services: an open-label, cluster-randomised controlled trial. Lancet Glob Health 2019;7:e366-75.

35 Yousafzai AK, Obradović J, Rasheed MA, et al. Effects of responsive stimulation and nutrition interventions on children's development and growth at age 4 years in a disadvantaged population in Pakistan: a longitudinal follow-up of a clusterrandomised factorial effectiveness trial. Lancet Glob Health 2016;4:e548-58.

36 Forsyth JE, Weaver KL, Maher K, et al. Sources of blood lead exposure in rural Bangladesh. Environ Sci Technol 2019;53:11429-36.

37 World Health Organization. Thinking healthy: a manual for psychological management or perinatal depression, 2015. Available: http://www.who.int/mental_health/maternal-child/thinking_healthy/ en/ [Accessed 8 Dec 2019]. 